\title{
Low Complexity Time-Concatenated Turbo Equalization for Block Transmission Without Guard Interval: Part 1-The Concept
}

\author{
Khoirul Anwar • Tad Matsumoto
}

Published online: 24 March 2012

(C) The Author(s) 2012. This article is published with open access at Springerlink.com

\begin{abstract}
This paper proposes a novel time-concatenated turbo equalization technique, chained turbo equalization (CHATUE), that allows block transmission systems to eliminate the guard interval (GI), while achieving excellent performance. The proposed CHATUE algorithm connects turbo equalizers neighboring in time, so that they exchange information about their inter-block-interference components in the form of a posteriori log-likelihood ratio. The latest version of the low complexity sub-optimal turbo equalization technique for block-wise single carrier transmission, frequency domain soft cancellation and minimum mean squared error, is fully exploited in developing the CHATUE algorithm. Results of extrinsic information transfer chart analysis as well as a series of bit-error rate (BER) simulations show that excellent performances can be achieved without imposing heavy computational burden in multipath-rich (quasi-static) block Rayleigh fading channels. It is shown that, if the information bit-rate is kept identical (because it may be unpreferable for the industry to change the frame structure), the CHATUE algorithm achieves lower BER than that with block transmission with GI, because lower rate (strong) code for error protection can be used by utilizing the time-duration made available by eliminating the GI. In addition, by combining the proposed structure with a simple rate-1 doped accumulator, further BER improvement exhibiting clear turbo cliff can be achieved. A sister paper (a Part-2 paper) applies the proposed CHATUE algorithm to single carrier frequency division multiple access systems Hui et al. (Wirel Pers Commun, 2011).
\end{abstract}

This work was supported in part by SANYO Electric Co., Ltd. and in part by the (Kinki) Mobile Wireless Center.

K. Anwar $(\varangle) \cdot$ T. Matsumoto

School of Information Science, Japan Advanced Institute of Science and Technology (JAIST),

1-1 Asahidai, Nomi, Ishikawa 923-1292, Japan

e-mail: anwar-k@jaist.ac.jp

T. Matsumoto

Center for Wireless Communication, University of Oulu, 90014 Oulu, Finland

e-mail: tadashi.matsumoto@ee.oulu.fi; matumoto@jaist.ac.jp 
Keywords Single carrier signaling - Guard interval (GI)/cyclic prefix (CP) - Turbo equalization · EXIT chart · Inter-block interference (IBI) · Inter-symbol interference (ISI) · Log-likelihood ratio (LLR) · Frequency domain · MMSE

\section{Introduction}

Block transmission systems using guard interval (GI), such as single carrier block transmission with frequency domain equalization [2] and orthogonal frequency division multiplexing (OFDM), both with cyclic prefix transmission (CP) [3,4], have been drawing much attention due to the robustness against channel's frequency selectivity. Quite recently, their mixed version, single carrier frequency division multiple access (SC-FDMA) [5] has been intensively investigated as a strong candidate for a wireless access scheme in next generation wireless communications systems, because of its flexibility in adjusting the parameters to radio air interface while satisfying each user's quality-of-service requirements.

It is common to the block transmission systems that the length of GI is assumed to be long enough compared to the length of the channel impulse response (CIR). However, when GI is shorter than the actual CIR length, the system will seriously suffer from inter-block interference (IBI) and inter-symbol interference (ISI), which often places bit error rate (BER) floor even at high signal-to-noise power ratio (SNR). To overcome this problem, and to reduce the interference due to insufficient GI length, several techniques have been presented, for example [6] where decision feedback equalization (DFE) is used to cancel the interference components from the past. Authors of [7] proposes block transmission without CP by assuming that interference from the past is perfectly cancelled, and that IBI from the future is equalized by using a Bayesian linear unbiased estimation (BLUE). Recently, authors of [8,9] have considered a past and future IBI cancellation technique by using a method called $\mathrm{CP}$ compensation (CPC) by utilizing the iterative structures. However, the BER performance can still be improved by considering information theoretic issues, especially the convergence property.

This paper proposes a new chain-structured time-concatenated frequency domain turbo equalization technique, chained turbo equalization (CHATUE), of which a block diagram is shown in Fig. 1. A goal of this paper is to show that the loss in the power and the spectrum efficiencies due to GI or CP transmission can be completely recovered by the proposed CHATUE algorithm if we can combine all energies spread in the past and the future blocks by exchanging interference knowledge between the blocks concatenated in the time domain. Another goal of this paper is to provide thorough convergence property analysis of the proposed and $[8,9]$ 's techniques.

To avoid unacceptable complexity, CHATUE algorithm exploits so called matrix J [7], with the aim of retrieving the circulant structure of the equivalent block-wise channel matrix; the use of the matrix $\mathbf{J}$ allows signal processing as much to stay in the frequency domain as in the latest version of a sub-optimal reduced-complexity turbo equalization technique for single carrier block-wise signaling, frequency domain soft cancellation and minimum mean square error (FD/SC-MMSE) filtering [10,11]; its required complexity is independent of the length of channel impulse response (CIR).

The CHATUE algorithm stores the received signal samples over several neighboring blocks in time, i.e. past, present, and future, and exchange a posteriori information of the symbols causing IBI between their connected equalizers. The a posteriori information is obtained from the decoders, and is used for soft cancellation of the IBI components. MMSE filtering is required to further suppress the residual interferences after the soft cancellation, which imposes the necessity of the inversion of the residual interference covariance matrices. 

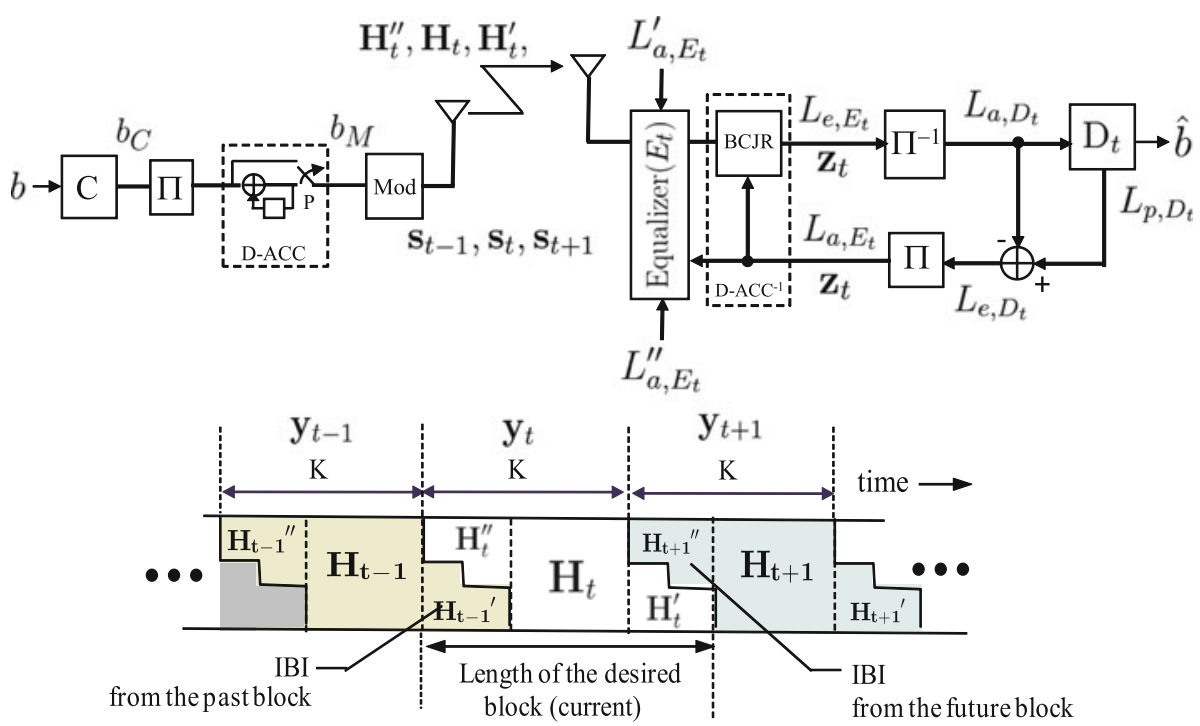

Fig. 1 System model for CHATUE algorithm for the $t$ th block and its equivalent channels

We have presented the original version of the CHATUE algorithm in part in [12]. In addition to the materials provided in [12], in this paper, we introduce further approximation to significantly reduce the complexity for the covariance matrix inversion. This paper also proposes the use of rate-1 doped accumulator (D-ACC) $[13,14]$ to achieve a clear turbo cliff in BER because of better matching of the extrinsic information transfer (EXIT) curves. Furthermore, it is also shown that, if the information per-block bit-rate is kept identical, ${ }^{1}$ the removal of GI/CP allows us to allocate more redundancy for error protection, resulting in better BER performance.

In this paper, CHATUE and its related techniques are proposed for single carrier block transmission (SCBT) systems. However, its extensions to other systems, e.g. OFDM and/or SC-FDMA systems are rather straightforward. A sister paper (a Part-2 paper) applies the proposed CHATUE algorithm to single carrier frequency division multiple access (SC-FDMA) systems [1].

The remainder of this paper is organized as follows. The system model is presented in Sect. 2. A full derivation of the CHATUE algorithm is presented in Sect. 3, and its approximated version for complexity reduction in Sect. 4. However, as described before, since the CHATUE algorithm largely depends on the latest version of FD/SC-MMSE, the derivations of some key equations, which can easily be derived from FD/SC-MMSE, are provided in Appendix for the completeness of the paper. Convergence property analysis is provided in Sect. 5 using EXIT chart. Section 6 presents results of a series of computer simulations conducted to evaluate performance of the proposed algorithm. Section 7 provides performance comparison with the existing technique. Finally, conclusion is given in Sect. 8 .

In this paper, the following notations are adopted. Scalars are expressed with standard text notation, vectors with bold lowercase, and matrices with bold uppercase. Past and future blocks relative to the current block are marked with $\bullet^{\prime}, \bullet$ ', respectively. An operator diag $\{\cdot\}$ with a vector argument denotes a diagonal matrix with vector elements on its diagonal com-

1 This is because it is preferable for the industry not to change the same frame structure. 
ponents. An estimate of variable is denoted by $\hat{\bullet}$ and a complex conjugation by $\bullet *$. Hermitian (transposed conjugate) of a matrix is indicated by $\bullet^{H}$. A $K \times K$ identity matrix is denoted as $\mathbf{I}_{K}$. The matrix transpose operator is denoted by $\bullet^{T}$. E[ [·] and $\operatorname{Pr}(\cdot)$ denote expectation and probability of their argument random variable, respectively. Unless specified, $L_{a}$ denotes $a$ priori LLR while $L_{p}$ a posteriori LLR.

\section{System Model}

A single-input single-output (SISO) system is assumed in this paper, however, its extension to multiple-input multiple-output (MIMO) systems is quite straightforward. The system model is depicted in Fig. 1. At the transmitter, information bits $b$ to be transmitted is transformed into a coded sequence $b_{C}$ by an encoder $C$, interleaved by a random interleaver $\Pi$, and in the case D-ACC is used, $b_{C}$ is further transformed to a doped-accumulated sequence $b_{M}$. The modulation symbol sequence to be transmitted at the $t$ th block timing is then given by

$$
\mathbf{s}_{t}=\left[s_{t}^{[0]}, s_{t}^{[1]}, \ldots, s_{t}^{[k]} \ldots s_{t}^{[K-1]}\right]^{T} \in \mathbb{C}^{K \times 1},
$$

where $[k]$ is the symbol index and with $K$ being the block length. The doping rate provides additional degree-of-freedom to achieve better matching in convergence property between CHATUE equalizer and decoder, which will be detailed in Sect. 5.

The block is then transmitted over a fading channel with frequency selectivity. Let $\mathbf{H}_{t-1}^{\prime}, \mathbf{H}_{t}$, and $\mathbf{H}^{\prime \prime}{ }_{t+1}$ denote the equivalent block-wise representations of the channel matrices corresponding to the past, the current and the future blocks, respectively, relative to the current block index $t$.

When CP is appended at the transmitter side (and eliminated it at the receiver side), the channel matrix $\mathbf{H}_{t}$ becomes circulant. However, in this paper, CP transmission is not assumed, for which the channel matrix for the current block has a structure, as

$$
\mathbf{H}_{t}=\left[\begin{array}{cccc}
h_{0}^{[0]} & & & 0 \\
\vdots & h_{0}^{[1]} & & \\
h_{L-1}^{[0]} & \vdots & \ddots & \\
& h_{L-1}^{[1]} & \vdots & h_{0}^{[K-1]} \\
& & \ddots & \vdots \\
0 & & & h_{L-1}^{[K-1]}
\end{array}\right]_{t} \in \mathbb{C}^{(K+L-1) \times K},
$$

with $L$ being the channel memory length. The channel matrices for the interference components from the past and the future blocks are given by

$$
\mathbf{H}_{t-1}^{\prime}=\left[\begin{array}{ccc}
h_{L-1}^{[K-L+1]} & \cdots & h_{1}^{[K-1]} \\
& \ddots & \vdots \\
& & h_{L-1}^{[K-1]} \\
& &
\end{array}\right]_{t-1} \in \mathbb{C}^{(K+L-1) \times K}
$$

and 


$$
\mathbf{H}_{t+1}^{\prime \prime}=\left[\begin{array}{ccc} 
& & 0 \\
h_{0}^{[0]} & & \\
\vdots & \ddots & \\
h_{L-2}^{[0]} & \cdots & h_{0}^{[L-2]}
\end{array}\right]_{t+1} \in \mathbb{C}^{(K+L-1) \times K},
$$

respectively, where $h_{0}^{[\cdot]}, \ldots, h_{L-1}^{[\cdot]}$ are the path gains. With quasi-static block Rayleigh fading assumption, where $h_{(\cdot)}^{[0]}=h_{(\cdot)}^{[1]}=\cdots=h_{(\cdot)}^{[K-1]}$, the matrix has a Toeplitz structure.

The algorithm assumes that the channel is known to the receiver. As shown in Fig. 1, the receiver has a CHATUE signal processing block, referred to as CHATUE equalizer $(E)$ later on, deinterleaver $\Pi^{-1}$, interleaver $\Pi$, and decoder $D$. The CHATUE equalizer receives four inputs, i.e. signal from the antenna, a priori LLR $L_{a, E_{t}}$ of the coded transmitted bits which is equivalent to the extrinsic LLR output of the decoder for the current block, a priori LLR $L_{a, E_{t}}^{\prime}$ provided by the past block decoder $D_{t-1}$, and a priori LLR $L_{a, E_{t}}^{\prime \prime}$ provided by the future block decoder $D_{t+1}$.

As mentioned before, the use of rate-1 D-ACC is considered in this paper. When D-ACC is used at the transmitter, the extrinsic LLR obtained by the the decoder, denoted by $D_{t}^{-1}$ is fed back to both CHATUE equalizer $E_{t}$ and the Bahl-Cocke-Jelinek-Raviv (BCJR) algorithm [15] used for the decoder of D-ACC, denoted by D-ACC ${ }^{-1}$, as shown in Fig. 1. Without D-ACC, on the other hand, $D_{t}^{-1}$, s extrinsic LLR is provided directly to $E_{t}$.

The received signal of the current block, $\mathbf{y}_{t}$, can be formulated as

$$
\mathbf{y}_{t}=\mathbf{H}_{t} \mathbf{s}_{t}+\mathbf{H}_{t-1}^{\prime} \mathbf{s}_{t-1}^{\prime}+\mathbf{H}_{t+1}^{\prime \prime} \mathbf{s}_{t+1}^{\prime \prime}+\mathbf{n} \in \mathbb{C}^{(K+L-1) \times 1},
$$

where $\mathbf{s}_{t}$ is the current block, $\mathbf{s}_{t-1}^{\prime}$ and $\mathbf{s}_{t+1}^{\prime \prime}$ are interference components from the past and future, respectively, and $\mathbf{n}$ is a zero mean complex additive white Gaussian noise (AWGN) vector with covariance $\mathbf{E}\left\{\mathbf{n n}^{H}\right\}=\sigma^{2} \mathbf{I}_{K+L-1} \cdot \sigma^{2}$ denotes the noise variance defined by received SNR with $\sigma^{2}=10^{-\mathbf{S N R}[\mathrm{dB}] / 10} . \mathbf{s}_{t-1}^{\prime}$ and $\mathbf{s}_{t+1}^{\prime \prime}$ are expressed as

$$
\mathbf{s}_{t-1}^{\prime}=\left[0, \ldots, 0, s_{t-1}^{[K-L+1]}, \ldots, s_{t-1}^{[K-1]}\right]^{T} \in \mathbb{C}^{K \times 1}
$$

and

$$
\mathbf{s}_{t+1}^{\prime \prime}=\left[s_{t+1}^{[0]}, \ldots, s_{t+1}^{[L-1]}, 0, \ldots, 0\right]^{T} \in \mathbb{C}^{K \times 1},
$$

respectively. $\mathbf{s}_{t-1}^{\prime}, \mathbf{s}_{t}$ and $\mathbf{s}_{t-1}^{\prime \prime}$ may be originated from the same user or different users. In the case the blocks are originated by different users, the system is equivalent to time division multiple access (TDMA).

The CHATUE equalizer for the $t$ th block receives three log-likelihood ratios (LLRs):

$$
L_{a, E_{t}}=\ln \frac{\operatorname{Pr}\left(b_{M_{t}}=0\right)}{\operatorname{Pr}\left(b_{M_{t}}=1\right)}
$$

for the ISI cancellation within the $t$ th block, and

$$
L_{a, E_{t}}^{\prime}=\ln \frac{\operatorname{Pr}\left(b_{M_{t-1}}=0\right)}{\operatorname{Pr}\left(b_{M_{t-1}}=1\right)}, L_{a, E_{t}}^{\prime \prime}=\ln \frac{\operatorname{Pr}\left(b_{M_{t+1}}=0\right)}{\operatorname{Pr}\left(b_{M_{t+1}}=1\right)}
$$

for the IBI cancellation from the past and the future blocks, respectively. Note that without DACC, $b_{M_{t-1}}=\Pi\left\{b_{C_{t-1}}\right\}, b_{M_{t}}=\Pi\left\{b_{C_{t}}\right\}, b_{M_{t+1}}=\Pi\left\{b_{C_{t+1}}\right\}$, where $\Pi\{\cdot\}$ is the interleaving function.

It should be noticed that $L_{a, E_{t}}$ is in the form of an extrinsic LLR due to the fact the iteration is performed between equalizer $E_{t}$ and decoder $D_{t}$, i.e. $L_{e, D_{t}}=L_{p, D_{t}}-L_{a, D_{t}}$, where $L_{p, D_{t}}$ 
and $L_{a, D_{t}}$ are a posteriori and a priori LLRs of the decoder, respectively. On the other hand, $L_{a, E_{t}}^{\prime \prime}=L_{p, D_{t+1}}$ and $L_{a, E_{t}}^{\prime}=L_{p, D_{t-1}}$ i.e. the a posteriori LLR is exchanged between the time-concatenated equalizers.

\section{Proposed CHATUE Algorithm}

The proposed CHATUE algorithm comprises the following three steps: (a) applying matrix $\mathbf{J}$ to obtain the circulant equivalent channel matrix, (b) the soft-cancellation of ISI and IBI, and (c) the residual interference cancellation which requires modifications on the original FD/SC-MMSE algorithm [11].

\subsection{Retrieval of Circularity}

To convert the Toeplitz matrix of the channel matrix $\mathbf{H}_{t}$ into a circulant matrix, we apply the matrix $\mathbf{J}$ as defined in [7]. With the channel matrix circularity retrieved by multiplying $\mathbf{J}$, we can best utilize the latest version of the FD/SC-MMSE algorithm. The matrix $\mathbf{J}$ is given by

$$
\mathbf{J}=\left[\begin{array}{c|c}
0_{(K-L+1) \times(L-1)} \\
\mathbf{I}_{(L-1) \times(L-1)}
\end{array} \mid \mathbf{I}_{K \times K}\right] \in \mathbb{C}^{K \times(K+L-1)} .
$$

Multiplying the matrix $\mathbf{J}$ by the received signal $\mathbf{y}_{t}$, the received block becomes

$$
\mathbf{r}_{t}=\mathbf{J y}_{t}=\mathbf{J} \mathbf{H}_{t} \mathbf{s}+\mathbf{J} \mathbf{H}_{t-1}^{\prime} \mathbf{s}_{t-1}^{\prime}+\mathbf{J H}_{t+1}^{\prime \prime} \mathbf{s}_{t+1}^{\prime \prime}+\mathbf{J n} \in \mathbb{C}^{K \times 1} .
$$

Notice that the current channel matrix $\mathbf{J H} t$ has a circulant structure, however, the matrices for the interferece components from neither past nor future satisfy the circulant properties because of their structures, as shown in (3) and (4).

\subsection{ISI and IBI Removal}

Because CP is not transmitted, the received signals suffers from IBI components from the past and the future blocks, while the ISI is caused by the multipath fading channel. IBI from the future block is inevitable if sampling continues until the end of the channel response to the last symbol in the current block, in order to collect the entire channel energy, as shown in the lower part of Fig. 1.

Figure 2 shows the detail of the chained structure, through which the exchange of the $a$ posteriori information from other decoders as well as of the extrinsic information from its own decoder takes place. The current ( $t$ th) CHATUE equalizer $E_{t}$, receives $L_{a, E_{t}}^{\prime}=L_{p, D_{t-1}}^{\prime}$ and $L_{a, E_{t}}^{\prime \prime}=L_{p, D_{t+1}}^{\prime \prime}$ from the decoders $\mathrm{D}_{t-1}$ and $\mathrm{D}_{t+1}$, respectively. The decoder $D_{t}^{-1}$ also provides a posteriori LLR $\mathbf{L}_{p, D_{t}}^{\prime}$ to the future block and $\mathbf{L}_{p, D_{t}}^{\prime \prime}$ to the past block. The $a$ priori LLR $L_{a, E_{t}}^{\prime}$ and $L_{a, E_{t}}^{\prime \prime}$ are used to remove the IBI from the past and the future blocks by calculating their soft estimates. Similarly, the a priori LLR $L_{a, E_{t}}\left(=L_{e, D_{t}}\right)$ is used to remove the symbol causing the ISI within the block by calculating their soft estimates in the same way as in the FD/SC-MMSE algorithm.

The IBI is actually caused by the last $L-1$ symbols in the past block as well as by the first $L-1$ symbols in the future block, as shown in (6) and (7). To remove ISI and IBI components, we construct soft replica of the receive signal given by (11), as

$$
\hat{\mathbf{r}}_{t}=\mathbf{J} \mathbf{H}_{t} \hat{\mathbf{s}}_{t}+\mathbf{J} \mathbf{H}_{t-1}^{\prime} \hat{\mathbf{s}}_{t-1}^{\prime}+\mathbf{J H}_{t+1}^{\prime \prime} \hat{\mathbf{s}}_{t+1}^{\prime \prime} \in \mathbb{C}^{K \times 1},
$$




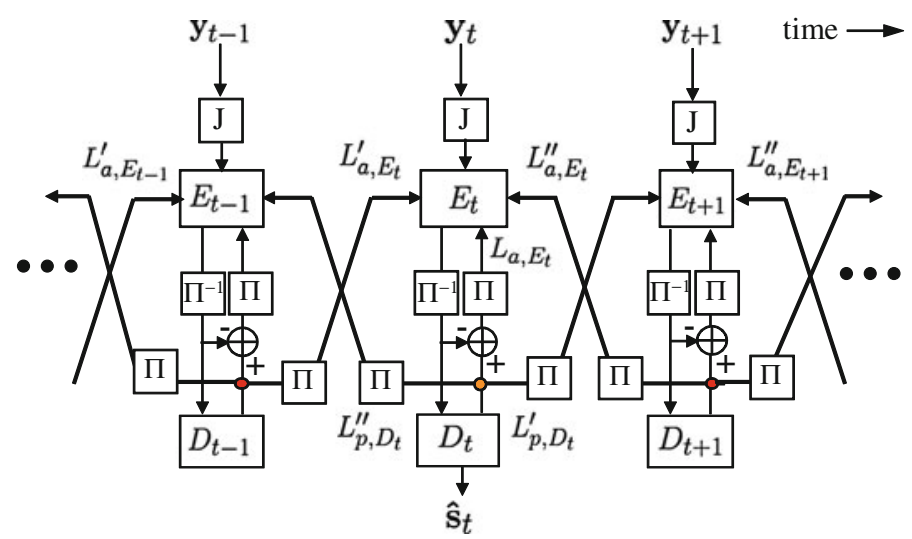

Fig. 2 LLR exchange between blocks $t-1, t$, and $t+1$ (for the case of without doped accumulator)

where $\hat{\mathbf{s}}_{t}=\left[\hat{s}_{t}^{[0]}, \hat{s}_{t}^{[1]}, \ldots, \hat{s}_{t}^{[K-1]}\right]^{T} \in \mathbb{C}^{K \times 1}, \hat{\mathbf{s}}_{t-1}^{\prime}=\left[0, \ldots, 0, \hat{s}_{t-1}^{[K-L+1]}, \ldots, \hat{s}_{t-1}^{[K-1]}\right]^{T} \in$ $\mathbb{C}^{K \times 1}$, and $\hat{\mathbf{s}}_{t+1}^{\prime \prime}=\left[\hat{s}_{t+1}^{[0]}, \ldots, \hat{s}_{t+1}^{[L-1]}, 0, \ldots, 0\right]^{T} \in \mathbb{C}^{K \times 1}$.

The $k$ th soft symbol estimate for binary phase shift keying (BPSK) is given by

$$
\hat{s}_{t}^{[k]}=E\left[s_{t}^{[k]}\right]=\sum_{s=-1,+1} s \cdot \operatorname{Pr}\left(s_{t}^{[k]}=s\right)=\tanh \left\{L_{a, E_{t}}\left[s_{t}^{[k]}\right] / 2\right\} .
$$

Similarly, $\hat{s}^{\prime[k]}{ }_{t-1}^{[k}=\tanh \left\{L_{a, E_{t}}^{\prime}\left[s_{t-1}^{[k]}\right] / 2\right\}$ and $\hat{s}_{t+1}^{\prime \prime}{ }_{t+1}^{[k]}=\tanh \left\{L_{a, E_{t}}^{\prime \prime}\left[s_{t+1}^{[k]}\right] / 2\right\}$, where $L_{a, E_{t}}^{\prime}$ and $L_{a, E_{t}}^{\prime \prime}$ denote the a priori LLR provided by the decoders for the past and the future blocks, respectively, in the form of the a posteriori LLR, for both of which the indexing $[k]$ corresponds to after deinterleaving.

Now, given the soft replica of the received signal $\mathbf{r}_{t}$ in (12), the interferences from the past, the current and the future blocks can be removed. To estimate the $k$ th symbol, we need to restore the $k$ th symbol, as

$$
\begin{aligned}
\hat{\mathbf{s}}_{t}^{[k]} & =\mathbf{r}_{t}-\hat{\mathbf{r}}_{t}+\mathbf{h}_{t}^{[k]} \hat{s}_{t}^{[k]} \\
& =\tilde{\mathbf{r}}_{t}+\mathbf{h}_{t}^{[k]} \hat{s}_{t}^{[k]} \in \mathbb{C}^{K \times 1},
\end{aligned}
$$

where $\mathbf{h}_{t}^{[k]}$ denotes the $k$ th column vector of the current channel matrix $\mathbf{J H} t$ with $\tilde{\mathbf{r}}=\mathbf{r}_{t}-\hat{\mathbf{r}}_{t}$ being the residual error remaining after the soft interference cancellation from the received signal $\mathbf{r}_{t}$.

\subsection{FD/SC-MMSE for CHATUE}

To reduce the residual interference, we apply adaptive filtering in the same way as in FD/SCMMSE. Slight modifications on the original FD/SC-MMSE have to be made due mainly to the use of the matrix $\mathbf{J}$ for the CP removal. However, FD/SC-MMSE is already well-known, and its details can be found in many literatures, such as $[10,11]$. Therefore, detailed derivation of FD/SC-MMSE for CHATUE is presented in Appendix, and only the final equations are provided in this section.

The final output vector $\mathbf{z}_{t}$ of the CHATUE algorithm for the $t$ th block is given by

$$
\mathbf{z}_{t}=\left(\mathbf{I}_{K}+\Gamma \mathbf{S}_{t}\right)^{-1}\left[\Gamma \hat{\mathbf{s}}_{t}+\mathbf{F}^{H} \Phi^{H} \mathbf{X}^{-1} \mathbf{F} \tilde{\mathbf{r}}_{t}\right] \in \mathbb{C}^{K \times 1},
$$


where $\mathbf{S}_{t}$ is the absolute value of soft estimate current symbols. $\Phi$ is the equivalent frequency domain channel matrix, while $\Gamma$ and $\mathbf{X}$ are the weights value that minimize the MMSE estimation error, as defined defined in the Appendix, and $\mathbf{F}$ is the discrete Fourier transform matrix.

To obtain the LLR of the equalizer output, we utilize the widely-used enough-accurate approximation, with which $\mathbf{z}_{t}$ can be expressed as being equivalent to a Gaussian channel output [13] as,

$$
\mathbf{z}_{t}=\mu_{t} \mathbf{s}_{t}+\mathbf{v}_{t} \in \mathbb{C}^{K \times 1}
$$

with

$$
\mu_{t}=\mathrm{E}\left[\mathbf{z}_{t} \cdot \mathbf{s}_{t}^{*}\right]=\frac{1}{K} \operatorname{tr}\left\{\Gamma\left(\mathbf{I}_{K}+\Gamma \mathbf{S}_{t}\right)^{-1}\right\}
$$

where $\mathbf{v}_{t}$ is the equivalent noise vector with variance being $\sigma_{t}^{2}=\mu_{t}\left(1-\mu_{t}\right)$. In (17), we used the approximation [10],

$$
\mathbf{S}_{t}=\operatorname{diag}\left\{\left|\hat{\mathbf{S}}_{t}\right|^{2}\right\} \approx \frac{1}{K} \sum_{k=1}^{K}\left|\hat{s}_{t}^{[k]}\right|^{2} \cdot \mathbf{I}_{K} \in \mathbb{C}^{K \times K} .
$$

Now, we can calculate the extrinsic LLR of the transmitted binary symbol by

$$
L_{e, E_{t}}\left[s_{t}^{[k]}\right]=\ln \frac{\operatorname{Pr}\left(z_{t}^{[k]} \mid s_{t}^{[k]}=+1\right)}{\operatorname{Pr}\left(z_{t}^{[k]} \mid s_{t}^{[k]}=-1\right)}=\frac{4 \Re\left(z_{t}^{[k]}\right)}{1-\mu_{t}},
$$

with $z_{t}^{[k]}$ being the $k$ th component of $\mathbf{z}_{t}$ and $\Re\left(z_{t}^{[k]}\right)$ denoting the real part of the complex $z_{t}^{[k]}$.

\section{Approximations}

As shown in (15), the complexity of the CHATUE algorithm is dominated by $\mathbf{X}^{-1}$. The complexity of the $\mathbf{X}^{-1}$ calculation can be reduced by taking into account the structure of $\mathbf{X}$. Recalling (34) in Appendix, we have

$$
\begin{aligned}
\mathbf{X}= & \Phi \mathbf{F} \Lambda \mathbf{F}^{H} \Phi^{H}+\mathbf{F} \mathbf{J} \mathbf{H}^{\prime} \Lambda^{\prime}\left(\mathbf{J H}^{\prime}\right)^{H} \mathbf{F}^{H}+\mathbf{F} \mathbf{J} \mathbf{H}^{\prime \prime} \Lambda^{\prime \prime}\left(\mathbf{J H}^{\prime \prime}\right)^{H} \mathbf{F}^{H} \\
& +\mathbf{F} \sigma^{2} \mathbf{J} \mathbf{J}^{H} \mathbf{F}^{H} \in \mathbb{C}^{K \times K},
\end{aligned}
$$

with which the shapes of each component matrix $\mathbf{X}$ are shown in the upper part of Fig. 3, where darker plots indicate larger values.

It can be observed from the upper part of Fig. 3 that the diagonal parts dominate in all the component matrices, even in the covariance matrices of the residual interference from the past and the future, $\mathbf{F} \mathbf{J} H^{\prime} \Lambda^{\prime}\left(\mathbf{J H}^{\prime}\right)^{H} \mathbf{F}^{H}$ and $\mathbf{F} \mathbf{J H}{ }^{\prime \prime} \Lambda^{\prime \prime}\left(\mathbf{J} H^{\prime \prime}\right)^{H} \mathbf{F}^{H}$, respectively. Considering the diagonal-dominant structure of those matrices, it is reasonable to approximate the matrix $\mathbf{X}$ by only using the diagonal components, with which the off-diagonal elements are replaced with zeros, while the main diagonal elements are replaced with the results of the trace operation $\frac{1}{K} \operatorname{tr}[\cdot]$.

Furthermore, because the well-known approximation $\mathbf{F} \Lambda \mathbf{F}^{H} \approx \frac{1}{K} \operatorname{tr}[\Lambda] \cdot \mathbf{I}_{K}$ is accurate when $K$ is large enough (e.g. $K=512$ )[10], the further approximation of $\Phi \mathbf{F} \Lambda \mathbf{F}^{H} \Phi^{H}=\Lambda$ 

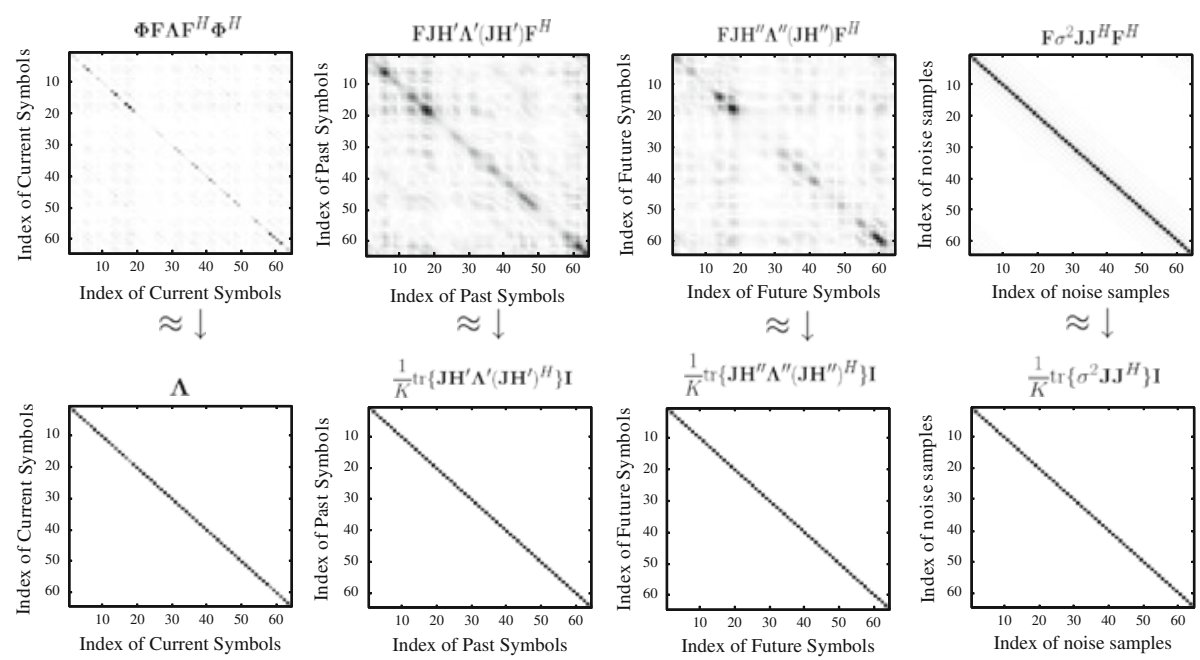

Fig. 3 Shapes of exact and approximated covariance matrix components of $\mathbf{X}$ at $I_{a, E_{t}}=I_{a, E_{t}}^{\prime}=I_{a, E_{t}}^{\prime \prime}=0.5$ and $K=64$

is reasonable in practice. Finally, with the approximation of (18), the computation of $\mathbf{X}$ can be reduced to

$$
\mathbf{X} \approx \Lambda+\frac{1}{K} \operatorname{tr}\left[\mathbf{J H}^{\prime} \Lambda^{\prime}\left(\mathbf{J H}^{\prime}\right)^{H}\right] \mathbf{I}_{K}+\frac{1}{K} \operatorname{tr}\left[\mathbf{J H}^{\prime \prime} \Lambda^{\prime \prime}\left(\mathbf{J H}^{\prime \prime}\right)^{H}\right] \mathbf{I}_{K}+\frac{\sigma^{2}}{K} \operatorname{tr}\left[\mathbf{J} \mathbf{J}^{H}\right] \mathbf{I}_{K} \in \mathbb{C}^{K \times K} .
$$

For each of the terms in (21), the shape is shown in the lower part of Fig. 3. Based on (20) and the approximation of (21), the computational burden for the matrix inversion, $\mathbf{X}^{-1}$, is negligible, because $\mathbf{X}$ is now diagonal.

With the technique described above, the computational complexity of the CHATUE algorithm is significantly reduced from $(28+K)(K \times K)+3 K$ complex additions-and-subtractions (ADDs-and-SUBs), $(30+K)(K \times K)+3 K$ complex multiplications (MULs), and $K \times K$ complex divisions (DIVs) to $14(K \times K)+5 K$ ADDs-and-SUBs, $20(K \times K)+2 K$ MULs and $K \times K$ DIVs.

\section{EXIT Chart Analysis}

\subsection{Without Doped Accumulator}

To evaluate the convergence property of the proposed CHATUE algorithm, the result of EXIT analysis is presented in this subsection. The sequences of the a posteriori LLRs provided by the decoders for the past and the future blocks are approximated as being Gaussian-distributed [13]. In the following, without loss of generality, we omit block index $t-1, t$, and $t+1$ (unless necessary) for the sake of simplicity in expression since the past can always be associated with $t-1$ and the future with $t+1$.

The receiver comprises two parts, the CHATUE equalizer and the decoder. As noted before, the CHATUE equalizer has three a priori LLR inputs, $L_{a, E}^{\prime}, L_{a, E}^{\prime}$ and $L_{a, E}^{\prime \prime}$, and one extrinsic LLR output, $L_{e, E}$, with which the EXIT function is expressed as 


$$
I_{e, E}=T_{E}\left(I_{a, E}, I_{a, E}^{\prime}, I_{a, E}^{\prime \prime}, \mathrm{SNR}\right),
$$

where $I_{e, E}$ denotes the mutual information between extrinsic LLR $L_{e, E}$ at the equalizer output and the interleaved and coded bits $b_{M}$, where as described before, without D-ACC $b_{M}=\Pi\left\{b_{C}\right\}$, as shown in Fig. 1. $I_{e, E}$ is given by

$$
I_{e, E}=I\left(L_{e, E} ; b_{M}\right)=\frac{1}{2} \sum_{m=-1,+1} \int_{-\infty}^{+\infty} \operatorname{Pr}\left(L_{e, E} \mid m\right) \cdot \log _{2} \frac{\operatorname{Pr}\left(L_{e, E} \mid m\right)}{\operatorname{Pr}\left(L_{e, E}\right)} d L_{e, E},
$$

where $\operatorname{Pr}\left(L_{e, E} \mid m\right)$ is the conditional probability of $L_{e, E}$ given $m=1-2 b_{M}=\{-1,+1\}$ [13].

Following (23), the a priori mutual information of the the equalizer is defined as $I_{a, E}=$ $I\left(L_{a, E} ; b_{M}\right), I_{a, E}^{\prime}=I\left(L_{a, E}^{\prime} ; b_{M}^{\prime}\right)$ and $I_{a, E}^{\prime \prime}=I\left(L_{a, E}^{\prime \prime} ; b_{M}^{\prime \prime}\right)$.

The decoder has one input, $L_{a, D}$, and one LLR output, $L_{e, D}$, which are independent of SNR because it has no connection to the channel. The decoder's EXIT function is expressed as

$$
I_{e, D}=T_{D}\left(I_{a, D}\right),
$$

where the extrinsic LLR is obtained as $L_{e, D}=L_{p, D}-L_{a, D}$. The mutual information $I_{e, D}$ and $I_{a, D}$ are defined as in (23) i.e. $I_{e, D}=I\left(L_{e, D} ; b_{C}\right)$ and $I_{a, D}=I\left(L_{a, D} ; b_{C}\right)$, where $b_{C}$ is the coded information bits as shown in Fig. 1.

Figure $4 \mathrm{a}$ shows the EXIT curves of the equalizer and the decoder for $E_{b} / N_{0}=4 \mathrm{~dB}$, where $E_{b} / N_{0}=\mathrm{SNR} \times 1 / \mathcal{R}$ with $\mathcal{R}$ being the channel coding rate. A half-rate non-systematic non-systematic non-recursive convolutional coding (NSNRCC) with the constraint length of 3 and a generator polynomial $G=[7,5]_{8}$ is considered. ${ }^{2}$ The trajectory of the mutual information exchange, obtained by the simulation is also shown in Fig. 4 a.

Since there are three LLR inputs and one LLR output, the EXIT function of the equalizer will be four-dimensional (4D). For the simplicity in description, in this section, we only plot the lower and upper bound EXIT curves. The lower bound curve is obtained by setting $I_{a, E}^{\prime}=I_{a, E}^{\prime \prime}=0$, which corresponds to the case where IBI remains not canceled, and the upper bound curve by setting $I_{a, E}^{\prime}=I_{a, E}^{\prime \prime}=1$, which to the case where IBI can be completely canceled. The EXIT curve of the decoder are drawn by measuring the histogram of the decoder output LLR, obtained by the BCJR algorithm [15].

It is found from the trajectory shown in Fig. 4a that with the mutual information feedback from the past and the future being zero, the EXIT curves intersect at a point indicated by A, which is far from the $I_{e, D}=1$ point. With the mutual information from the past and the future block being one, the EXIT curves intersect at the point B which is higher than A.

The trajectory confirms that the LLRs feedback from the neighboring blocks helps the equalizer EXIT curve be lifted up, by which intersection point moves from A to B, thereby the performance is improved. However, because the intersection point B is still relatively far from $I_{e, D}=1$, the infinitesimal BER, $\operatorname{Pr}(\hat{b} \neq b) \rightarrow 0$, is unachievable in practice.

\subsection{With Doped Accumulator}

Since without D-ACC the convergence tunnel between the equalizer and decoder does not suddenly open until the $(1,1)$ point as shown in Fig. 4a even with $I_{a, E}^{\prime}=I_{a, E}^{\prime \prime}=1$, we can not expect a clear turbo cliff to happen with the parameter settings described in Sect. 5.1.

\footnotetext{
2 It should be noted here that there was a minor mistake in Fig. 5 of [12]. The encoder was erroneously written as CC-4[17,15] in [12], but it is actually CC-3[7,5]. Furthermore, the decoder's EXIT chart presented in [12] is for $\mathrm{CC}-3[7,5]$.
} 


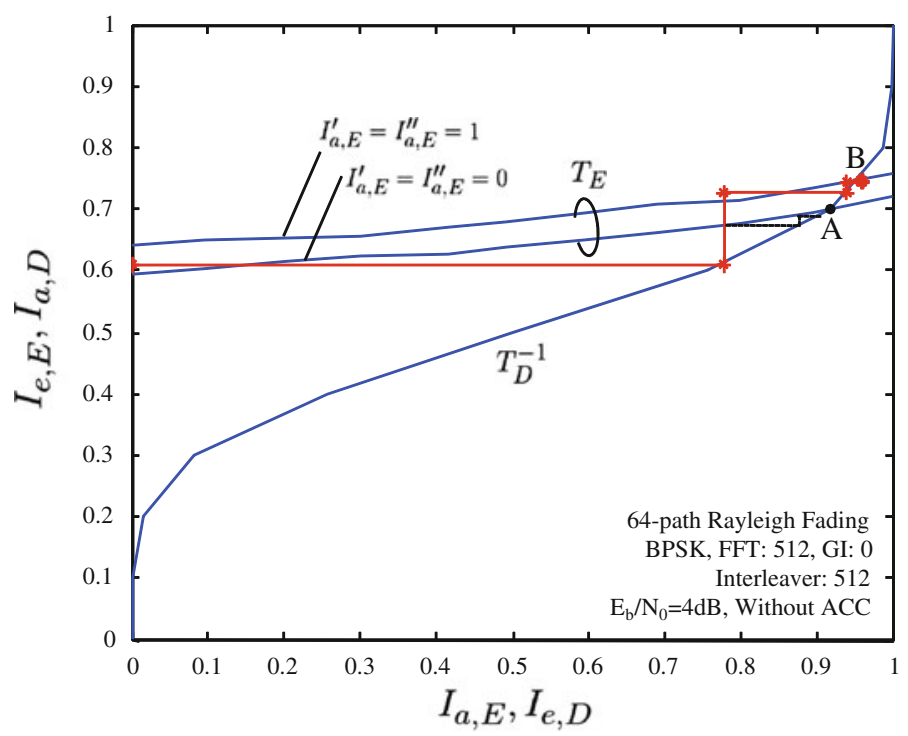

(a)

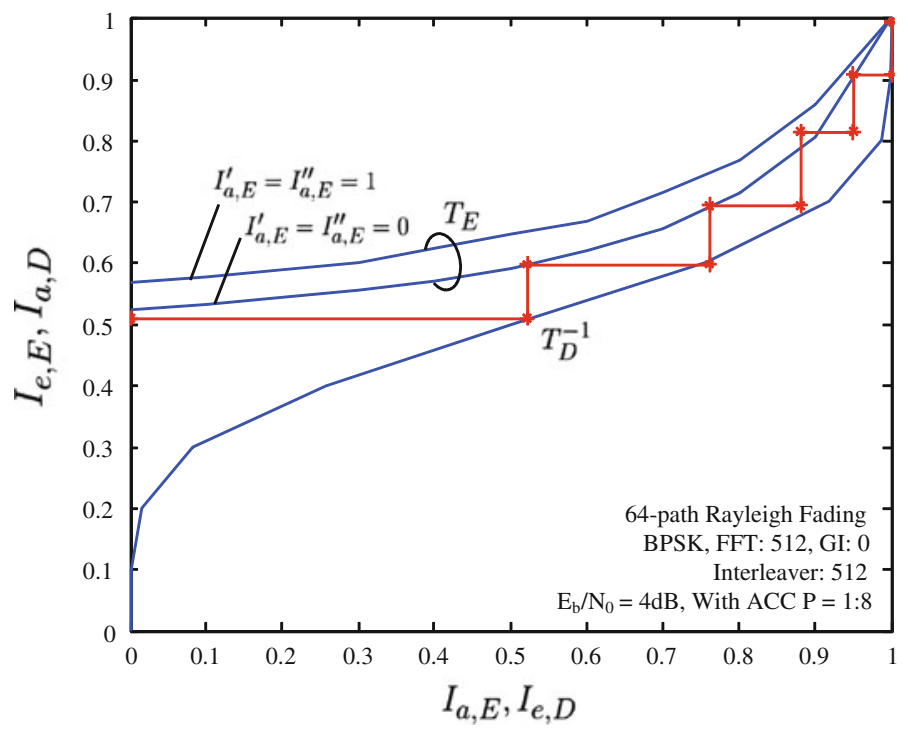

(b)

Fig. 4 EXIT analysis of the CHATUE algorithm without and with doped accumulator. a Without doped accumulator, $\mathbf{b}$ with doping rate $P=8$

Furthermore, the trajectory in Fig. 4a also illustrate that no more significant iteration gain is obtained after 3 iterations, even with $I_{a, E}^{\prime}=I_{a, E}^{\prime \prime}=1$.

To solve this problem, as shown in Fig. 1, we introduce a slight modification on the structure by utilizing D-ACC $[14,16]$ to bend the equalizer EXIT curve so that the convergence tunnel opens up to the $(1,1)$ mutual information point. In addition, the use of D-ACC allows 
us to change the shape of the equalizer's EXIT curve to match with the EXIT curve of the decoder. It should be noted that such precise curve fitting is not possible only with non-doped ACC, but it is made possible by adjusting the doping rate $P$ in D-ACC.

With D-ACC, the equalizer ${ }^{3}$ and decoder EXIT functions have similar shape, and thereby the convergence tunnel suddenly opens until the $(1,1)$ point, and the gap between the equalizer and decoder becomes smaller, resulting in less information-rate loss[17].

Since the D-ACC is a memory-one systematic recursive convolutional coding (SRCC), the BCJR algorithm can be used for the decoder, $\mathrm{D}-\mathrm{ACC}^{-1}$, without imposing heavy computational burden. The D-ACC, which is located before the BPSK modulator, has a doping rate of $P$, indicating that every $P$ th information bit is replaced by the accumulated bit. Since $\mathrm{D}-\mathrm{ACC}$ is rate-1 encoder, the total code-rate is unchanged.

EXIT curves of the CHATUE algorithm with the D-ACC is shown in Fig. 4b, where other parameters are the same as that used in Fig. 4a. The trajectory shows that the $(1,1)$ mutual information point can be reached without having intersection. Furthermore, the slope of their EXIT curves are similar, and thereby a clear turbo cliff is expected.

\section{Performances Evaluation}

A series of computer simulations was conducted to verify the performances of the CHATUE algorithm. Single carrier block transmission with $\mathrm{CP}$ (SCCP) and without CP (SCBT) were assumed. The binary information sequence is convolutionally encoded using NSNRCC with generator polynomial of $G=[7,5]_{8}$. BPSK modulation was used. We assumed 64-path equal average power Rayleigh fading channel of which path gains are perfectly known to the receiver. Random interleavers with length of 512 were used, and the maximum number of iterations was 9. The Log-MAP BCJR algorithm was used for the decoders $D_{t}^{-1}, D_{t-1}^{-1}, D_{t+1}^{-1}$ as well as for D-ACC ${ }^{-1}$.

BER was measured with the same parameters as in the EXIT analysis provided in the Sect. 5. All performance results shown below were measured based on the reduced complexity CHATUE algorithm with approximations provided in Sect. 4. The total power, totalling over all the path, was normalized to be unity, i.e.

$$
\sum_{\ell=0}^{L-1}\left|h_{\ell}\right|^{2}=1
$$

\subsection{Advantage of CP Removal}

The effectiveness of CP removal is verified in this subsection. The BER performance was evaluated by setting $I_{a, E}^{\prime}=I_{a, E}^{\prime \prime}=\{0.0,0.5,0.75,1.0\}$. The x-axis represents $E_{b} / N_{0}$, where bandwidth expansion factor due to the $\mathrm{CP}$ transmission is taken into account for fair comparison as $E_{b} / N_{0}=\mathrm{SNR} \times 1 / \mathcal{R} \times 1 / M \times(K+L) / K$, where the modulation multiplicity $M=1$ for BPSK.

Let the code-rate of CHATUE be denoted by $\mathcal{R}_{\mathrm{CHATUE}}$, and that of SCCP by $\mathcal{R}_{\mathrm{SCCP}}$. By keeping the block length of CHATUE and SCCP identical so that $K_{\mathrm{CHATUE}}=K_{\mathrm{SCCP}}+L$, the advantage of CHATUE is obvious, because

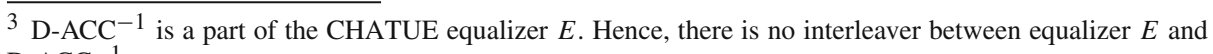
D-ACC ${ }^{-1}$. 


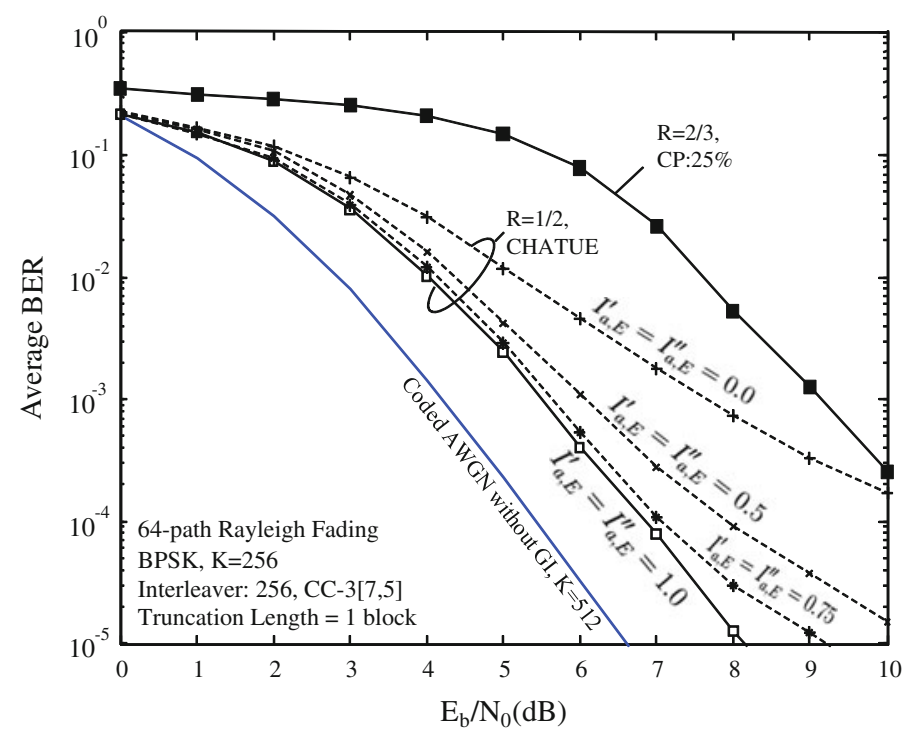

Fig. 5 Advantages of removing GI

$$
\frac{N}{\mathcal{R}_{\text {CHATUE }}}=\frac{N}{\mathcal{R}_{\text {SCPP }}}+L,
$$

where $N$ is the length of the information in bits. Assuming that the code rate is adjusted only by puncturing from the same mother code, BER performance of CHATUE can be made obviously better than SCCP because the redundant part, used for the CP transmission, can be utilized for further error protection, resulting in $\mathcal{R}_{\text {CHATUE }}<\mathcal{R}_{\text {SCCP }}$.

The BER performance with SCCP were evaluated for (a) CP ratio $=25 \%$ (for $K=256$ ) and (b) $\mathrm{CP}$ ratio $=12.5 \%$ (for $K=512$ ). The results are shown in Fig. 5, where the $\mathrm{CP}$ ratios were changed while keeping the CP length $L=64$ constant and the block length being $K=256$ and $K=512$; with the parameter setting described above, the code-rate of CHATUE is $\mathcal{R}_{\text {CHATUE }}=1 / 2(=128 / 256$ and 256/512), while the code-rates for SCCP are $\mathcal{R}_{\mathrm{SCCP}}=256 /(512-64)=4 / 7($ for CP ratio $=12.5 \%)$ and $\mathcal{R}_{\mathrm{SCCP}}=128 /(256-64)=$ $2 / 3$ (for CP ratio $=25 \%$ ).

It is found from Fig. 5 that the improvement by CHATUE is about $3.7 \mathrm{~dB}$ at BER $=$ $2 \times 10^{-4}$ when the CP ratio is $25 \%$. $^{4}$ The gain with CHATUE tends to be smaller with smaller CP ratio; with $\mathrm{CP}$ ratio $=12.5 \%$, the gain is about $0.3 \mathrm{~dB}$, because the difference in the code-rate is smaller $\left(\mathcal{R}_{\mathrm{CHATUE}}=1 / 2\right.$ and $\left.\mathcal{R}_{\mathrm{SCCP}}=4 / 7\right)$. The BER improvement described above verify the advantage of block transmission without GI, which is made possible if the interference from the past and future blocks can be eliminated by using the time-concatenated turbo equalization.

\subsection{Truncation Length}

In the previous section, the purpose of the simulations was to evaluate the BER performance by assuming $I_{a, E}^{\prime}=I_{a, E}^{\prime \prime}=\{0.0,0.5,0.75,1.0\}$. In practice, however, because of the latency

4 The code rate $\mathcal{R}_{\mathrm{SCCP}}=2 / 3$ is obtained by puncturing of an NSNRCC with pattern of [10;11] of a convolutional code with a generator matrix of $G=[7,5]_{8}$. 
requirement, the chain has to be truncated with a certain number $T=2 B+1$, where $B$ is the number of the considered neighboring blocks on the both sides in time as shown in Fig. 2. In this subsection, the impact of the chain truncation is evaluated.

The BER performance comparison was evaluated for $T=3$ and $T=5$. As a reference, BER of SCCP in one-path static (non-fading) AWGN channel without CP transmission is plotted. The mutual information was set at $I_{a, E_{t-1}}^{\prime}=I_{a, E_{t+1}}^{\prime \prime}=0$ (for $T=3$ ) and $I_{a, E_{t-2}}^{\prime}=I_{a, E_{t+2}}^{\prime \prime}=0$ for $(T=5)$. The results ${ }^{5}$ confirm that the performances corresponding to the truncation lengths of $T=3$ and $T=5$ are virtually identical.

Furthermore, the simulation results showed that the difference in BER between the two cases, one with $T=3$ truncated chain and the other with perfect mutual information assumption, $I_{a, E}=I_{a, E}^{\prime}=I_{a, E}^{\prime \prime}=1$, as discussed in Sect. 6.1, is negligible. Therefore, it can be concluded here that 3-block truncation is enough for the CHATUE algorithm.

Figure 6a shows the BER performance of the CHATUE algorithm with 3-block truncation length. Uncoded BPSK BER in flat Rayleigh fading channel is plotted as the upper bound BER, and the coded BER of SCBT without CP in 1-path static (non-fading) AWGN channel is also plotted as a reference for the lower bound BER. It is found that without D-ACC, the BER performance of CHATUE is improved through iterations and finally converge after 3 iterations with the difference from the lower bound of about $0.5 \mathrm{~dB}$ in $E_{b} / N_{0}$. It is important to note that that improvement by CHATUE without D-ACC is insignificant after 3 iterations, even 9 iterations were performed in the simulation, which is due mainly to the mismatch between the EXIT curves of the CHATUE equalizer and the decoder.

\subsection{Performance with Doped Accumulator}

BER performance with D-ACC is shown in Fig. 6b, where the same parameters were assumed as in the EXIT analysis discussed in Sect. 5 . The lower bound was evaluated by setting $T=1$ and $I_{a, E}^{\prime}=I_{a, E}^{\prime \prime}=1$, while the rest of the curves are with $T=3$ truncated chain with $I_{a, E_{t-1}}^{\prime}=I_{a, E_{t+1}}^{\prime \prime}=0$. As shown by the EXIT chart in Fig. 4b, the D-ACC should help the CHATUE algorithm achieve the $(1,1)$ mutual informations point. With the D-ACC, the two EXIT curve have a similar curvature, and hence clear turbo cliff are expected to happen.

As shown in Fig. 6b, the CHATUE performance can significantly be improved by the D-ACC, achieving a clear turbo cliff at $E_{b} / N_{0}=4.5 \mathrm{~dB}$ after 9 iterations. The performance with 3-block truncation length is only about $0.2 \mathrm{~dB}$ away from the lower bound BER, which is obtained by setting $I_{a, E}=I_{a, E}^{\prime}=I_{a, E}^{\prime \prime}=1$ with a $P=8 \mathrm{D}$-ACC.

\subsection{Impact of Channel Variation}

Up to this point, we have assumed quasi-static block Rayleigh fading, where the channel variation in a block due to the Doppler spread is neglected. However, in practice fading variation in a block still remains, and hence evaluating the channel variation in a block is of significant importance, when practical implementation is considered. The channel coefficients $h_{(\cdot)}^{[\cdot]}$ vary according to the normalized Doppler frequency, $f_{d} T_{s}$, normalized to the single carrier symbol duration $T_{s}$. It should be noted here that in single carrier signaling systems, frame/block contains $K$ symbols, while in OFDM systems [4], one block is defined as one OFDM symbol. Therefore, when $f_{d} T_{s}$ is normalized over the block/frame duration, we can write the new normalized Doppler shift as

$$
f_{d} T_{s}^{\prime}=K \times f_{d} T_{s}
$$

5 The BER performance evaluation result for $T=5$ is not shown in this paper because of the space limitation. 


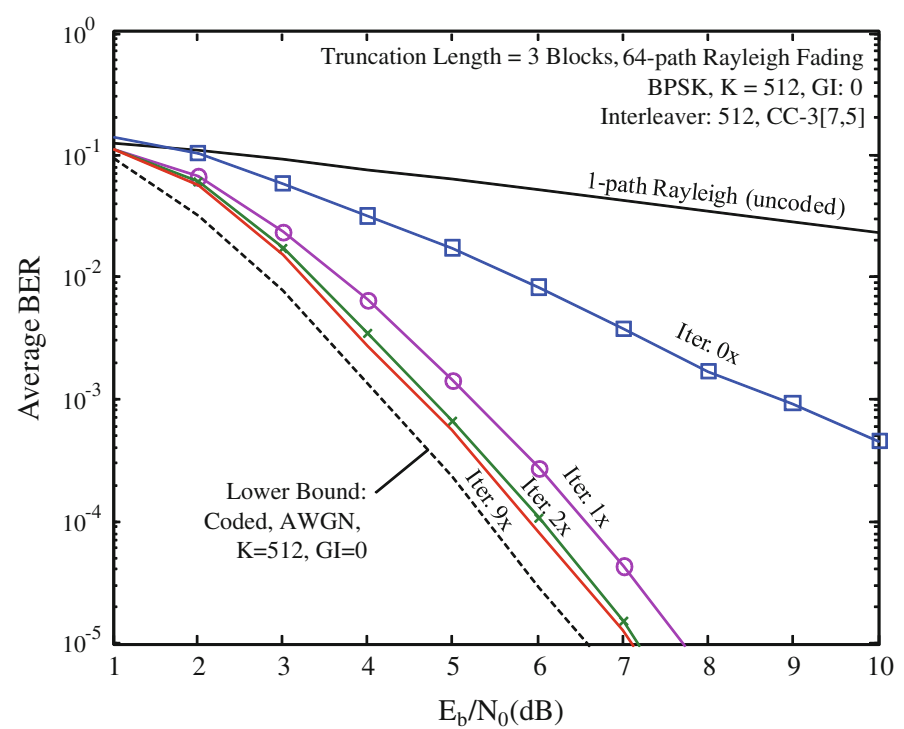

(a)

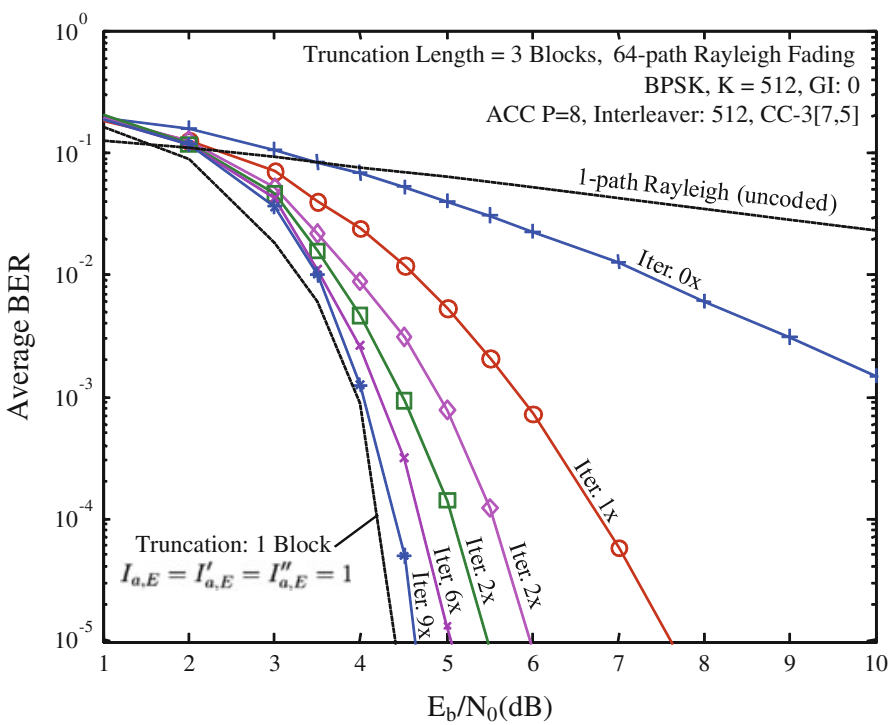

(b)

Fig. 6 BER performance of the CHATUE algorithm. a BER performance without doped accumulator, $\mathbf{b}$ BER performances with doped accumulator

where $K$ is the block length. However, for the general expression, through out the paper, we use $f_{d} T_{s}$ and evaluate the performance through computer simulations.

Since the block-wise processing for FD/SC-MMSE requires the channel to be constant within one block, resulting in the equivalent channel matrix $\mathbf{H}_{t}$ in (2) having Toeplitz structure with the constant elements column-wise. However, in practice, because of the time-varying nature of the channel, each column vector in the channel matrix should not necessarily be 


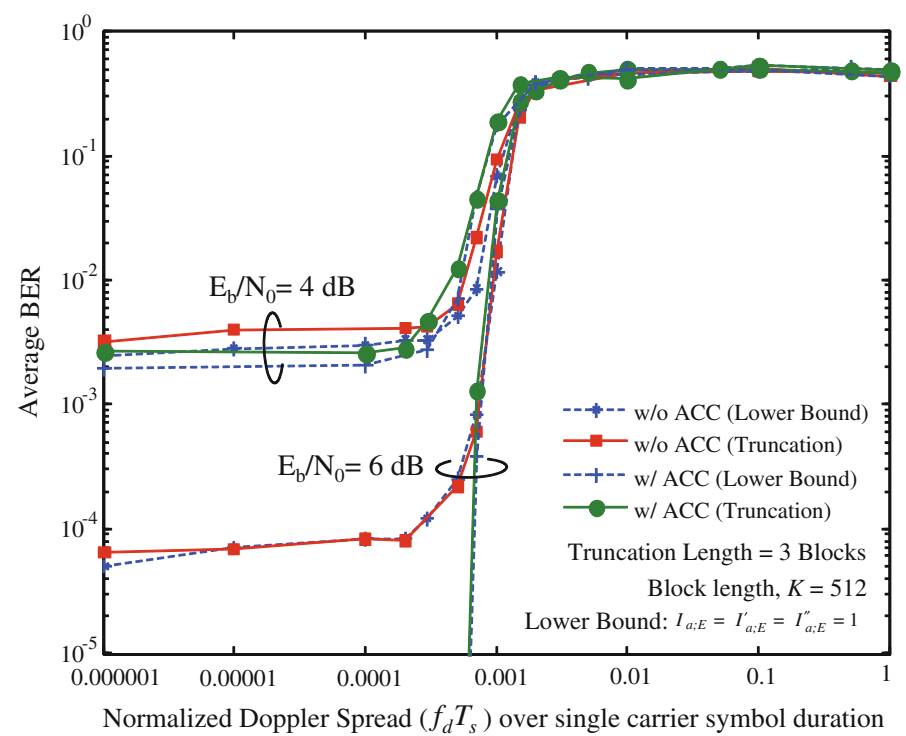

Fig. 7 CHATUE algorithm with and without D-ACC in the presence of channel variation, where $f_{d} T_{S}$ is normalized over the single carrier symbol duration (equivalent to $K f_{d} T_{s}$ in OFDM systems)

the down-shifted version of the first column vector, and the elements of each column vector gradually change due to the channel variation.

Let $\overline{\mathbf{H}}$ be the average of the time-varying channel matrix, having element $\bar{h}_{\ell}$, defined as

$$
\bar{h}_{\ell}=\frac{1}{K} \sum_{k=0}^{K-1} h_{\ell}^{[k]},
$$

where $h_{\ell}^{[k]}$ is the $\ell$ th path gain at the symbol index [k], as defined in (2). Simulations were conducted using the approximation of (28), where the time-varying matrix $\mathbf{H}_{t}$ was replaced by its average, as

$$
\mathbf{H}_{t}=\left[\begin{array}{cccc}
h_{0}^{[0]} & & & 0 \\
\vdots & h_{0}^{[1]} & & \\
h_{L-1}^{[0]} & \vdots & \ddots & \\
& h_{L-1}^{[1]} & \vdots & h_{0}^{[K-1]} \\
& & \ddots & \vdots \\
0 & & & h_{L-1}^{[K-1]}
\end{array}\right]_{t}\left[\begin{array}{cccc}
\bar{h}_{0} & & & 0 \\
\vdots & \bar{h}_{0} & & \\
\bar{h}_{L-1} & \vdots & \ddots & \\
& \bar{h}_{L-1} & \vdots & \bar{h}_{0} \\
& & \ddots & \vdots \\
0 & & & \bar{h}_{L-1}
\end{array}\right]_{t}=\overline{\mathbf{H}}_{t} \in \mathbb{C}^{(K+L-1) \times K}
$$

The time-varying matrix $\mathbf{H}_{t-1}$ and $\mathbf{H}_{t+1}$ were also assumed to be represented by their timeaverage $\overline{\mathbf{H}}_{t-1}$ and $\overline{\mathbf{H}}_{t+1}$, respectively.

With a truncation length of 3 blocks, each having $K=512$ symbols, BER performance was evaluated versus the normalized Doppler $f_{d} T_{s}$ as a parameter. The results are plotted in Fig. 7 for $E_{b} / N_{0}=4 \mathrm{~dB}$ and $6 \mathrm{~dB}$, where the Jakes model shown in [18] was assumed as the Doppler spectrum. 
It is observed that the gap in BER between the lower bound BER and that with 3-block truncation length is negligible when $E_{b} / N_{0}=6 \mathrm{~dB}$. Hence, a conclusion is drawn that with $K=512$ and other parameters described in Sect. 6, the normalized Doppler frequency $f_{d} T_{s}<0.001$ is required so that the block (quasi-static) fading assumption does not make significant degradation in performance.

In fact, with $K$ being the number of the symbols in each block, $K \times f_{d} T_{S}<K \times 0.001$ is very reasonable for block transmission system of any kinds, including OFDM [4], where $K T_{S}$ is equivalent to the OFDM symbol duration. In the case of SCBT, to eliminate the influence of excessively large Doppler spread, a long block has to be split into relatively short sub-blocks, and they are transmitted in succession by inserting GI/CP, with which decrease in spectrum efficiency is unavoidable. With the CHATUE algorithm, if the value of $K$ is chosen properly, it can eliminate the influence of excessively high Doppler frequency without sacrificing the spectrum efficiency, because it does not require the GI/CP transmission between the sub-blocks.

\section{Performance Comparison with Other Techniques}

To assess effectiveness of the proposed CHATUE algorithm, this subsection makes a performance comparison with the CP compensation (CPC) technique presented in [8,9] for block transmission without GI. According to the definition provided in [8,9], ISI is caused only by $\mathrm{CP}$ symbols $\mathbf{s}_{j}^{C P}$, where $j$ denotes the current block. The objective of the CPC technique is, to the best of our knowledge, only to cancel IBI because of the fact that ISI $=\tilde{\mathbf{H}}_{1^{\prime}} \mathbf{s}_{j}^{C P}=0$, even when CP is not transmitted. ${ }^{6}$ However, in frequency selective channels, the ISI lasts over the duration of the channel memory length and it may not be canceled by the CPC. The CHATUE algorithm, on the other hand, soft-cancels both ISI and IBI, and their residuals further suppressed by MMSE, with the help of a priori feedback. Hence, the CHATUE achieves better performance than CPC.

BER performances with the CHATUE and CPC algorithms were evaluated via computer simulations for quantitative comparison. For fair comparison, CHATUE without D-ACC was used. The assumed channel in this section follows 21-path Hilly Terrain model, also used in $[8,9]$. The $\mathcal{R}=1 / 2$ memory 6 NSNRCC with $G=[171,133]_{8}$ was used. The block length $K=64$ was assumed, which is the same as the interleaver length. It should be emphasized that all parameters setting are identical to that used in $[8,9]$.

\subsection{EXIT Chart Comparison}

The performance superiority of the proposed CHATUE over CPC can be recognized by EXIT chart analysis presented in this sub-section. Figure 8 shows the EXIT curves of the proposed CHATUE (without D-ACC) and CPC. It can be observed that the EXIT curve with CPC is almost flat, where intersections with the decoder EXIT curve happen at the point $\mathrm{C}$ with $I_{a, E}^{\prime}=I_{a, E}^{\prime \prime}=0$, and D with $I_{a, E}^{\prime}=I_{a, E}^{\prime \prime}=1$.

The proposed CHATUE has decay, which results in intersection with the decoder EXIT curve at a point closer to the $(1,1)$ mutual information point, the points $E$ with $I_{a, E}^{\prime}=I_{a, E}^{\prime \prime}=0$ and $\mathrm{F}$ with $I_{a, E}^{\prime}=I_{a, E}^{\prime \prime}=1$, even with the same $E_{b} / N_{0}$, as shown in Fig. 8. This indicates that $\mathrm{CPC}$ requires higher $E_{b} / N_{0}$ to achieve points $\mathrm{E}$ or $\mathrm{F}$ than with CHATUE.

$\overline{6}$ The notations exactly follow [8] and [9]. 


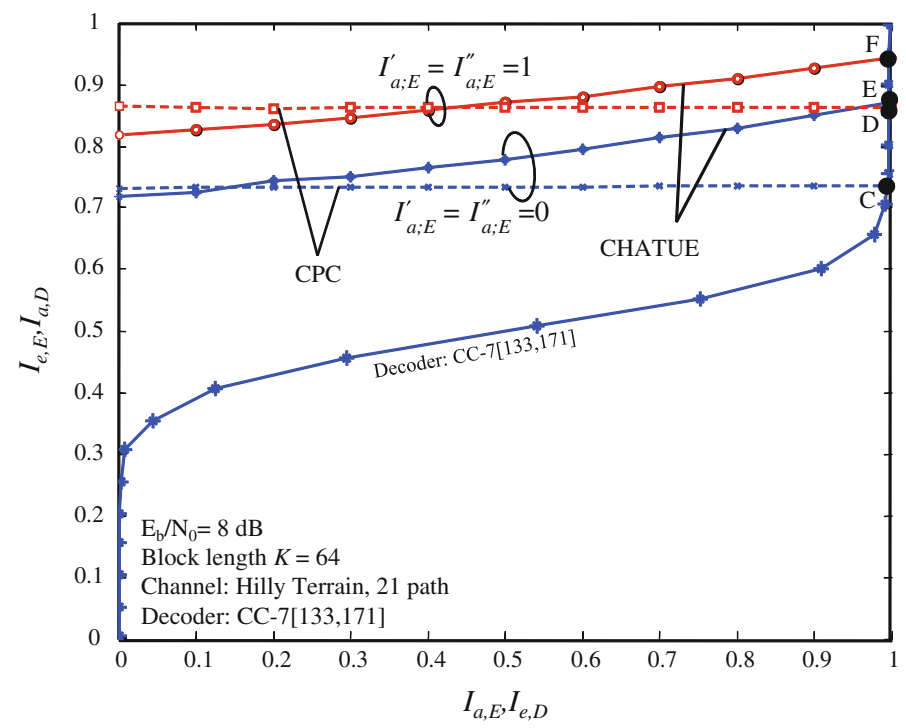

Fig. 8 EXIT chart of CHATUE algorithm (without D-ACC) and CPC $[8,9]$

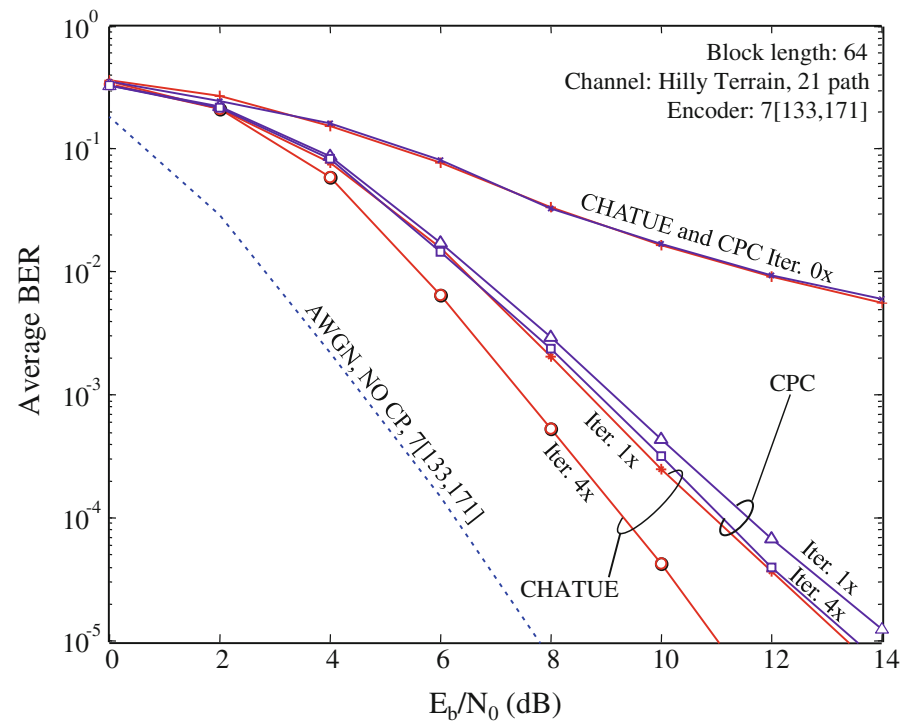

Fig. 9 BER performance of CHATUE algorithm and CPC $[8,9]$

\subsection{BER Performance Comparison}

With CPC, iterations do not improve BER performance significantly, because of the flat CPC EXIT curve. On the other hand, iterations within the current block provide larger gains with the proposed CHATUE algorithm and the performance is roughly $2.5 \mathrm{~dB}$ better than CPC at a BER range of $10^{-4}-10^{-5}$.

It should be noted here that the BER with CHATUE shown in Fig. 9 is worse than that in Fig. 6a. This is because of the difference in simulation parameters which are indicated in the 
Table 1 Computational complexity of the proposed CHATUE and CPC

\begin{tabular}{lll}
\hline Operations & CPC $[8,9]$ & The proposed CHATUE \\
\hline Addition and subtraction & $(6+K)(K \times K)+3 K$ & $14(K \times K)+5 K$ \\
Multiplications & $(7+K)(K \times K)+4 K$ & $20(K \times K)+2 K$ \\
Divisions & $(K \times K)+K$ & $K \times K$ \\
\hline
\end{tabular}

figures. It should be noted that those parameters in Fig. 9 are exactly the same as those used in [8] and [9] for fair comparison.

\subsection{Computational Complexity Comparison}

The computational complexity is dominated by the covariance matrix inversion for both CPC and CHATUE. Table 1 compares the computational complexity required for the CPC and the CHATUE algorithms. It can be observed from the table that the computational complexity of CHATUE algorithm is about order of $K^{2}$, while CPC is $K^{3}$.

\section{Conclusion}

A novel chained turbo equalization (CHATUE) technique as well as its complexity reduced version has been proposed in this paper as a framework for block transmission without GI/CP. Since its flexibility in structure of signal processing, its extension to OFDM and/or SC-FDMA systems is rather straightforward. The IBI and ISI components are removed by utilizing the knowledge exchange of interferences between the neighboring blocks. The EXIT chart analysis and the BER performance evaluation have confirmed that the proposed CHATUE algorithm, when applied to SCBT, achieves excellent performance and is capable of eliminating the IBI and ISI effectively, resulting in only about $0.5 \mathrm{~dB}$ away at BER $=10^{-4}$ from the lower bound. Further improvement of up to $2.7 \mathrm{~dB}$ in $E_{b} / N_{0}$ can be achieved by using a doped accumulator. It has also been confirmed that the impact of channel variation is negligible under normalized Doppler spread $f_{d} T_{s}<0.001$ following the Jakes' model.

Open Access This article is distributed under the terms of the Creative Commons Attribution License which permits any use, distribution, and reproduction in any medium, provided the original author(s) and the source are credited.

\section{Appendix: Derivation of the CHATUE Algorithm}

The MMSE filter $\mathbf{w}(k)$ is determined such that $\mathbf{w}(k)=\arg \underset{\mathbf{w}^{H}(k)}{\min }\left|\mathbf{w}^{H}(k) \hat{\mathbf{s}}(k)-s(k)\right|^{2}$, where the solution of weight vector $\mathbf{w}(k)$ is found to be

$$
\begin{aligned}
\mathbf{w}(k)= & \left(\mathrm{E}\left[\tilde{r} \tilde{r}^{H}\right]+\mathbf{h}(k)|\hat{\mathbf{s}}(k)|^{2} \mathbf{h}^{H}(k)\right)^{-1} \mathbf{h}(k) \\
= & \left(\mathbf{J H} \mathbf{\Lambda}(\mathbf{J H})^{H}+\mathbf{J H}^{\prime} \mathbf{\Lambda}^{\prime}\left(\mathbf{J H}^{\prime}\right)^{H}+\mathbf{J H}^{\prime \prime} \boldsymbol{\Lambda}^{\prime \prime}\left(\mathbf{J H}^{\prime \prime}\right)^{H}+\sigma^{2} \mathbf{J} \mathbf{J}^{H}\right. \\
& \left.+\mathbf{h}(k)|\hat{\mathbf{s}}(k)|^{2} \mathbf{h}^{H}(k)\right)^{-1} \mathbf{h}(k) \\
= & \left(\boldsymbol{\Sigma}+\mathbf{h}(k)|\hat{\mathbf{s}}(k)|^{2} \mathbf{h}^{H}(k)\right)^{-1} \mathbf{h}(k) \in \mathbb{C}^{K \times 1} .
\end{aligned}
$$


Since the interleaver is random enough, it is reasonable to assume that the soft symbol $\hat{\mathbf{s}^{\prime}}, \hat{\mathbf{s}}$ and $\hat{\mathbf{s}^{\prime \prime}}$ are uncorrelated, resulting in symbol-level covariance matrix of the ISI component remaining in the current block being diagonal, as

$$
\boldsymbol{\Lambda}=\operatorname{diag}\left\{\mathrm{E}\left[|\hat{\mathbf{s}}|^{2}\right]-|\hat{\mathbf{s}}|^{2}\right\}, \boldsymbol{\Lambda}^{\prime}=\operatorname{diag}\left\{\mathrm{E}\left[\left|\hat{\mathbf{s}}^{\prime}\right|^{2}\right]-\left|\hat{\mathbf{s}}^{\prime}\right|^{2}\right\}, \mathbf{\Lambda}^{\prime \prime}=\operatorname{diag}\left\{\mathrm{E}\left[\left|\hat{\mathbf{s}}^{\prime \prime}\right|^{2}\right]-\left|\hat{\mathbf{s}}^{\prime \prime}\right|^{2}\right\} .
$$

For BPSK modulation, (32) becomes $\boldsymbol{\Lambda}=\operatorname{diag}\left\{1-|\hat{s}(0)|^{2}, \ldots, 1-|\hat{s}(K-1)|^{2}\right\}, \boldsymbol{\Lambda}^{\prime}=$ $\operatorname{diag}\left\{0 \cdots 0,1-\left|\hat{s}^{\prime}(K-L+1)\right|^{2}, \cdots, 1-\left|\hat{s}^{\prime}(K-1)\right|^{2}\right\}, \boldsymbol{\Lambda}^{\prime \prime}=\operatorname{diag}\left\{1-\left|\hat{s}^{\prime \prime}(0)\right|^{2}, \ldots, 1-\right.$ $\left.\left|\hat{s}^{\prime \prime}(L-1)\right|^{2}, 0 \cdots 0\right\}$. With the estimate (14), the output of FD/SC-MMSE $z(k)=\mathbf{w}^{H}(k) \hat{\mathbf{s}}=$ $\mathbf{w}^{H}(k)(\tilde{\mathbf{r}}+\mathbf{h}(k) \hat{s}(k))$ becomes

$$
z(k)=\left(1+\gamma(k)|\hat{s}(k)|^{2}\right)^{-1} \mathbf{h}^{H}(k) \boldsymbol{\Sigma}^{-1}(\tilde{\mathbf{r}}(k)+\mathbf{h}(k) \hat{s}(k)),
$$

where $\gamma(k)=\mathbf{h}^{H}(k) \boldsymbol{\Sigma}^{-1} \mathbf{h}(k)$. By sorting all elements of $z(k)$ into a vector $\mathbf{z}$, the block wise expression of (33) is $\mathbf{z}=\left(\mathbf{I}_{K}+\Gamma \mathbf{S}\right)^{-1}\left[\Gamma \hat{\mathbf{s}}+\mathbf{H}^{H} \mathbf{J}^{H} \boldsymbol{\Sigma}^{-1} \tilde{\mathbf{r}}\right] \in \mathbb{C}^{K \times 1}$, where $\Gamma=$ $\operatorname{diag}\left[\mathbf{H}^{H} \mathbf{J}^{H} \boldsymbol{\Sigma}^{-1} \mathbf{J H}\right] \in \mathbb{C}^{K \times K}$ is a block-wise expression of $\gamma(k)$ in (33) and $\mathbf{S}=\operatorname{diag}\left[|\hat{\mathbf{s}}|^{2}\right]$.

Because the equalization is performed in the frequency domain, the beneficial property of circulant matrix $\mathbf{J H}$, which is $\mathbf{J H}=\mathbf{F}^{H} \Phi \mathbf{F}$, can be utilized to reduce the computational complexity of equalization processing since $\Phi=\mathbf{F} \mathbf{J H F}{ }^{H}$ is a diagonal matrix. As a consequence, $\Sigma$ in (31) can be expressed as $\Sigma=\mathbf{F}^{H} \Phi \mathbf{F} \boldsymbol{\Lambda} \mathbf{F}^{H} \Phi^{H} \mathbf{F}+\mathbf{J H}^{\prime} \boldsymbol{\Lambda}^{\prime}\left(\mathbf{J H}^{\prime}\right)^{H}+$ $\mathbf{J H}^{\prime \prime} \boldsymbol{\Lambda}^{\prime \prime}\left(\mathbf{J H}^{\prime \prime}\right)^{H}+\sigma^{2} \mathbf{J} \mathbf{J}^{H} \in \mathbb{C}^{K \times K}$, of which the frequency domain equivalent expression is

$$
\mathbf{X}=\mathbf{F} \boldsymbol{\Sigma} \mathbf{F}^{H} \in \mathbb{C}^{K \times K} .
$$

The final output of the CHATUE algorithm is given by $\mathbf{z}=\left(\mathbf{I}_{K}+\boldsymbol{\Gamma S}\right)^{-1}[\boldsymbol{\Gamma} \hat{\mathbf{s}}+$ $\left.\mathbf{F}^{H} \Phi^{H} \mathbf{X}^{-1} \mathbf{F} \tilde{\mathbf{r}}\right] \in \mathbb{C}^{K \times 1} . \mathbf{z}$ for the $t$ th block is shown as $\mathbf{z}_{t}$ in (15).

\section{References}

1. Hui, Z., Anwar, K., \& Matsumoto, T. (Sept. 2011). Low complexity time-concatenated turbo equalization for block transmission without guard interval: Part 2-application to SC-FDMA. Wireless Personal Communications. doi:10.1007/s11277-011-0409-1.

2. Sari, G. K. H., \& Jeanclaude, J. (Feb. 1995) Transmission techniques for digital terrestrial TV broadcasting. IEEE Communications Magazine, 100-109.

3. Weinstein, S. B., \& Ebert, P. M. (Oct. 1971). Data transmission by frequency-division multiplexing using discrete Fourier transform. IEEE Transactions on Communications, 19(5), 628-634.

4. Prasad, R., \& Nee, V. (2000). OFDM for wireless multimedia wireless communications. Boston, MA: Artech House.

5. Myung, H. G., Lim, J., \& Goodman, D. J. (2006). Single carrier FDMA for uplink wireless transmission. IEEE Vehicular Technology Magazine, 1(3), 30-38.

6. Suyama, S., Suzuki, H., \& Fukawa, K. (April 2003). An OFDM receiver employing the low-complexity turbo equalization in multipath environments with delay difference grater than the guard interval. In IEEE VTC2003-Spring (pp. 632-636).

7. Wang, D., Pan, Z., You, X., Kyu, C. H., Jang, J. B., \& Ha J. (Nov. 2008). Low-complexity turbo equalization for single-carrier systems without cyclic prefix. In IEEE ICCS'08, Guangzhou (pp. 1091-1095).

8. Lee, H., Lee, Y., \& Park, H. (2010). An efficient CP compensation for sc-fde with insufficient $\mathrm{cp}$ symbols. IEEE Communications Letters, 14(6), 548-550.

9. Lee, H., Lee, Y., Ann, K., \& Park, H. (May 2010) Interference cancellation for single carrier frequency domain equalizer without cyclic prefix. In IEEE VTC2010-Spring, Taiwan.

10. Kansanen, K., \& Matsumoto, T. (2003). A computationally efficient MIMO turbo equalizer. In IEEE vehicular technology conference (Vol. 1, pp. 277-281). Korea.

11. Kansanen, K., \& Matsumoto, T. (2007). An analytical method for MMSE MIMO turbo equalizer EXIT chart computation. IEEE Transactions on Wireless Communications, 6(1), 59-63.

12. Anwar, K., Hui, Z., \& Matsumoto, T. (May 2010). Chained turbo equalization for block transmission without guard interval. In IEEE VTC2010-Spring, Taiwan. 
13. ten Brink, S. (2001). Convergence behavior of iteratively decoded parallel concatenated codes. IEEE Transactions on Communications, 49, 1727-1737.

14. Pfletschinger, S., \& Sanzi, F. (2006). Error floor removal for bit-interleaved coded modulation with iterative detection. IEEE Transactions on Wireless Communications, 5(11), 3174-3181.

15. Bahl, L., Cocke, J., Jelinek, F., \& Raviv, J. (1974). Optimal decoding of linear codes for minimizing symbol error rate. IEEE Transactions on Information Theory, IT-20(2), 284-287.

16. ten Brink, S. (2000). Rate one-half code for approaching Shannon limit by $0.1 \mathrm{~dB}$. IEE Electronics Letters, 36, 1293-1294.

17. Asikhmin, A., Kramer, G., \& ten Brink, S. (Nov. 2004). Extrinsic information transfer functions: Model and erasure channel properties. IEEE Transactions on Information Theory, 50(11), 2657-2673.

18. Pop, M. F., \& Beaulieu, N. C. (2001). Limitations of sum-of-sinusoids fading channel simulators. IEEE Transactions on Communications, 49(4), 699-708.

\section{Author Biographies}

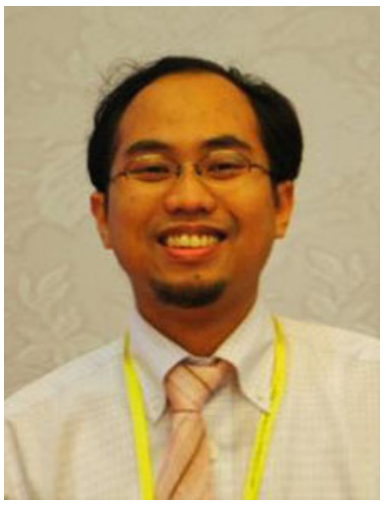

Khoirul Anwar graduated (cum laude) from the department of Electrical Engineering, Institut Teknologi Bandung (ITB), Bandung, Indonesia in 2000. He received Master and Doctor Degrees from Graduate School of Information Science, Nara Institute of Science and Technology (NAIST) in 2005 and 2008, respectively. Since then, he has been enrolled as an assistant professor at the same university until August 2008. He received best student paper award from the IEEE Radio and Wireless Symposium 2006, California-USA, Best Paper of ISA 2007, Kyoto, Japan in 2007. Since September 2008, he is with the School of Information Science, Japan Advanced Institute of Science and Technology (JAIST) as an assistant professor. His research interests are network information theory, error control coding, iterative decoding and signal processing for wireless communications.

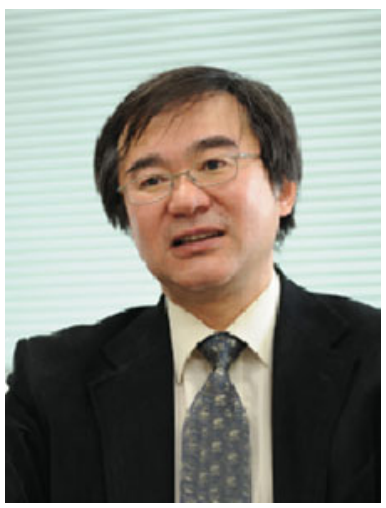

Tad Matsumoto received his B.S., M.S., and Ph.D. degrees from Keio University, Yokohama, Japan, in 1978, 1980, and 1991, respectively, all in electrical engineering. He joined Nippon Telegraph and Telephone Corporation (NTT) in April 1980. Since he engaged in NTT, he was involved in a lot of research and development projects, all for mobile wireless communications systems. In July 1992, he transferred to NTT DoCoMo, where he researched Code-Division Multiple-Access techniques for Mobile Communication Systems. In April 1994, he transferred to NTT America, where he served as a Senior Technical Advisor of a joint project between NTT and NEXTEL Communications. In March 1996, he returned to NTT DoCoMo, where he served as a Head of the Radio Signal Processing Laboratory until August of 2001; He worked on adaptive signal processing, multiple-input multiple-output turbo signal detection, interference cancellation, and space-time coding techniques for broadband mobile communications. In March 2002, he moved to University of Oulu, Finland, where he served as a Professor at Centre for Wireless Communications. In 2006, he served as a Visiting Professor at Ilmenau University of Technology, Ilmenau, Germany, funded by the German MERCATOR Visiting Professorship Program. Since April 2007, he has been serving as a Professor at Japan Advanced Institute of Science and Technology (JAIST), Japan, while also keeping the position at University of Oulu. T. Matsumoto has been appointed as a Finland Distinguished Professor for a period from January 2008 to December 2012, funded by the Finnish National Technology Agency (Tekes) and Finnish Academy, under which he preserves the rights to participate in and apply to European and Finnish national projects. He is a recipient of IEEE VTS Outstanding Service Award (2001), Nokia Foundation Visiting Fellow Scholarship Award (2002), IEEE Japan Council Award for Distinguished Service to the Society (2006), IEEE Vehicular Technology Society James R. Evans Avant Garde Award (2006), and Thuringia State Research Award for Advanced Applied Science (2006), 2007 Best Paper Award of Institute of Electrical, Communication, and Information Engineers of Japan (2008), and Telecom System Technology Award. 